\title{
Applying EVM to Satellite on Ground and In-Orbit Testing - Better Data in Less Time
}

\author{
Dr. Robert Peters* \\ Stellar Solutions, 250 Cambridge Ave., Palo Alto, CA 94306 \\ Elizabeth-Klein Lebbink and Victor Lee \\ Boeing Integrated Defense Systems, Network and Space Systems, El Segundo, CA \\ Josh Model, Robert Wezalis, Dr. John Taylor \\ Lincoln Laboratories, 244 Wood Street Lexington, MA 02420-9185
}

\begin{abstract}
Using Error Vector Magnitude (EVM) in satellite integration and test allows rapid verification of the Bit Error Rate (BER) performance of a satellite link and is particularly well suited to measurement of low bit rate satellite links where it can result in a major reduction in test time (about 3 weeks per satellite for the GOES satellites during ground test) and can provide diagnostic information. Empirical techniques developed to predict BER performance from EVM measurements and lessons learned about applying these techniques during GOES N, $\mathrm{O}$, and $\mathrm{P}$ integration and test and post launch testing, are discussed.
\end{abstract}

\section{Introduction}

The Geosynchronous Operational Environmental Satellite (GOES) N, O, and P satellites, while primarily weather and science satellites, also have eight specialized communication links. Each link is unique and varies in data rate from $100 \mathrm{bps}$ to $2.66 \mathrm{Mbps}$. Two links use uncoded RF links to transmit data generated on the satellite. Unlike BER measurements, EVM allows the BER to be determined with actual data, which might be different from a Pseudo Random (PN) code test pattern. These characteristics make EVM measurements the best method to verify link performance without taking the link out of service to test with a test pattern.

EVM is a well established technique used in measuring performance of cellular communication systems $^{1,2}$. The application of EVM to satellites is not new, as it has been used to characterize unit level $^{3}$ and system level performance in Europe and in the US, specifically at NASA ${ }^{4}$. Consequently, commercial vector signal analyzers (VSAs) to measure EVM are readily available from major electronic companies including Agilent, Tektronics, and Rhode\&Schwarz.

EVM is simply the standard deviation of the points about an average value of a constellation point (Figure 1). The constellation plot provides information on the link quality and, unlike a BER measurement, provides information about the factors impairing the link that can be used as diagnostic information about the degradations that limit performance. EVM can measure performance on an

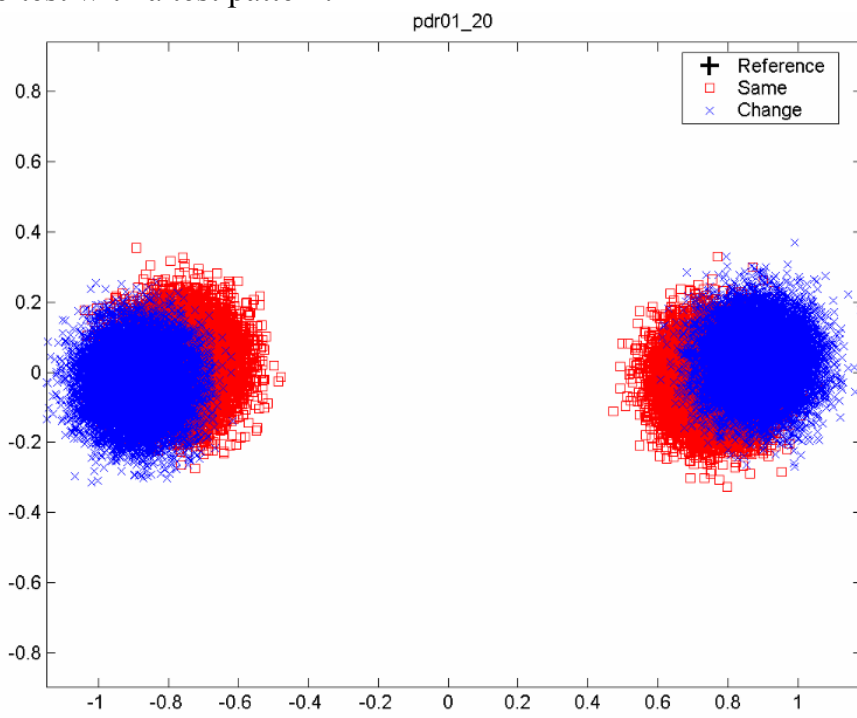

Figure 1 Measured EVM for BPSK. EVM shows difference between same bit transmitted (squares) and a change in bit (x's)

${ }^{*}$ Goddard Space Flight Center, Greenbelt, Maryland 20771 
operational link that contains all impairments up to the EVM measurement point (which can be RF or IF). A second major advantage of EVM over BER tests is that knowledge of the original bit sequence is not needed. This was particularly important for the GOES N, O, \& P satellites as they have science data generated on-board the satellite that is transmitted without coding to the ground. Figure 1 also shows that the constellation plot is sensitive to its history due to intersymbol interference (due to the VSA itself, channel filter mismatch, ringing, or other effects). If the bit is the same as the previous bit, its constellation point is different than if the bit has changed. This give rise to the two blob effect discussed later.

Some authors have said that EVM cannot be used to predict BER. For example Johnson (2001) states, "EVM is a summation of effects. It does not differentiate between random effects which add geometrically and possess probability densities, from distortion effects which add directly and are deterministic. EVM is useful because its characteristics can tell us something about the nature of the problem or where the signal is degraded, but it can not be directly related to BER, the acid test for the network operator." ${ }^{5}$ Here we show that EVM can be used to determine BER using an empirical calibration. Once a calibration curve is obtained, a very quick EVM measurement (typically 60,000 samples) can be used to determine BER to very low bit rates. This was especially useful for low data rate signals, such as the $1 \mathrm{kbps}$ and lower rates on the GOES satellites. EVM can be used to predict BER only if the changes are due to changes in the AWGN (additive white Gaussian noise). If the change in BER is due to another mechanism, perhaps an increase in phase noise, the technique developed here will show that a change has occurred, but its prediction of the BER associated with the change may not be accurate.

\section{Relating EVM to BER}

While in principle the BER could be calculated solely from the standard deviation of the EVM, such calculations do not agree with BER measurements in part because they do not take into account modem impairments. It was necessary to develop equations to relate BER (or probability of error, Pet) to EVM as shown below ${ }^{6}$. The equations developed have two variables $\alpha$ and

$\beta$ which are adjusted to give a best fit to the measured BER curve (bit error rate vs. signal to noise ratio). This measured curve is obtained from simultaneous BER and EVM measurements (or separate measurements under the same Pet $=\frac{1}{2} \operatorname{erfo}(1-\alpha E V M) \sqrt{\left.\frac{\left(-\frac{1}{10} S N R\right)+\frac{\beta E V M}{\pi}}{10}\right)}$ conditions). The number of samples taken determines the accuracy of the measurement. About 60,000 measurements provide an EVM value with a standard deviation accurate to about $0.4 \%$. (Please refer to the companion paper $^{7}$ for the derivation of the formula shown here and for more information on development of the equations, variables and measurement methods).

Figure 2 shows a measured BER curve and the best fit curve, where $\alpha$ and $\beta$ were adjusted for best fit. This agreement is typical for the GOES measurements. Another useful aspect of EVM is demonstrated in Figure 2. The performance at extremely low BERs can be extrapolated backward. The link shown in Figure 2 is a 25 kbps link. What would normally be a very time consuming measurement, determining BER to $10^{-8}$ requiring approximately $10^{9}$ bits or about 10 hours can be done in minutes with EVM.

\section{Intersymbol interference and the "Two-Blob Effect"}

Non-ideality in the VSA, the RF modulator or filtering can result in inter-symbol interference (ISI) ${ }^{8}$. For example most VSA's have a stock set of matching filters (e.g. Raised Cosine, Root Raised Cosine), and if none truly match the spacecraft hardware then ISI will result, as shown in figures 1 and 3.

Using a simple EVM calculation would result in a distorted EVM value as it would imply an oval shape. Since we are using EVM to calibrate the AWGN response of a system, we need to remove the effect of the ISI in order to isolate the AWGN component of EVM. This was accomplished by calculating separate EVM statistics for symbols 


\section{EMWIN}

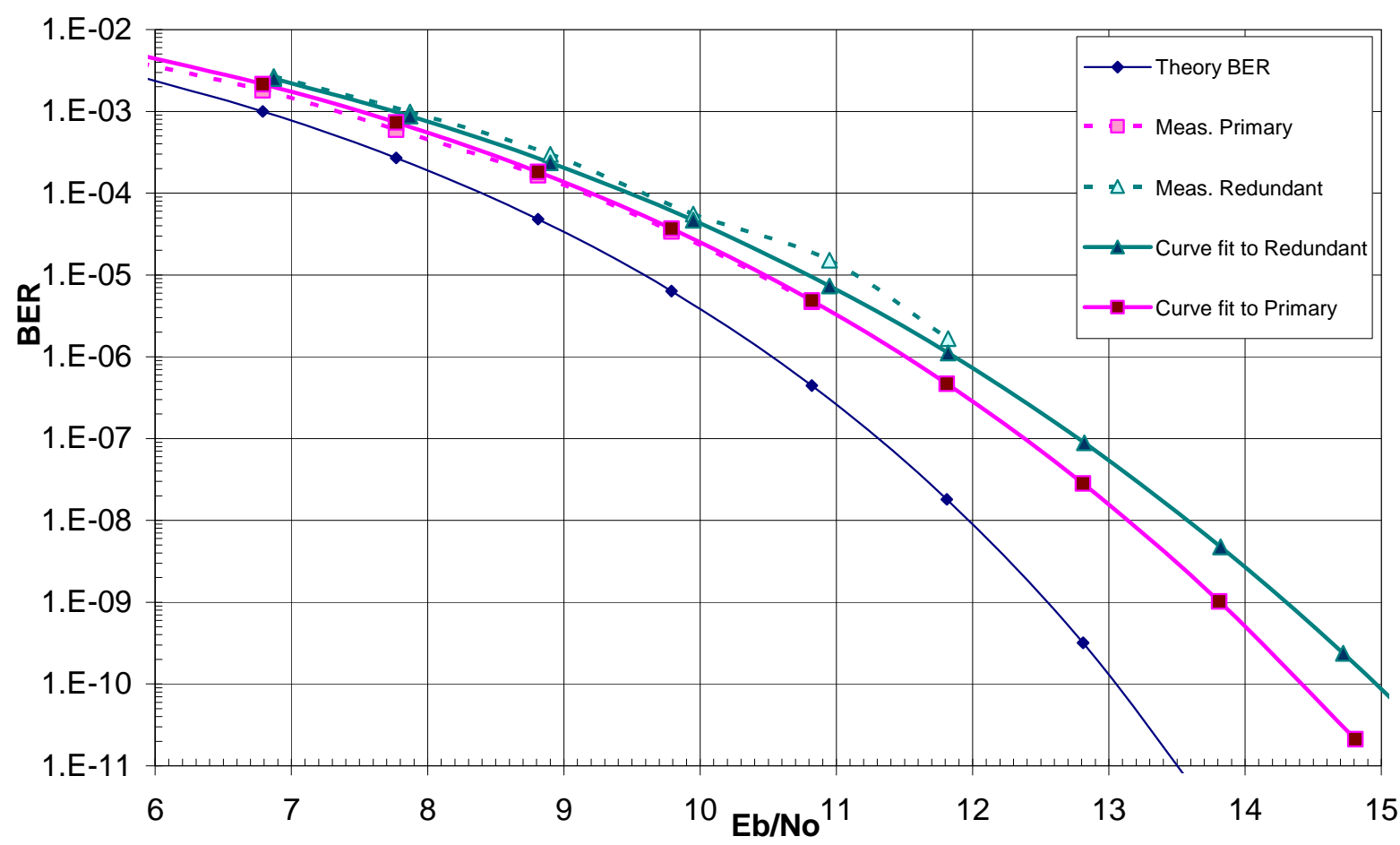

Figure 2 Measured BER and curve fitted BER

sampled immediately after a transition, and symbols sampled without a change in logical value as shown in Figure 3. This only removes channel memory effects due to the previous symbol,

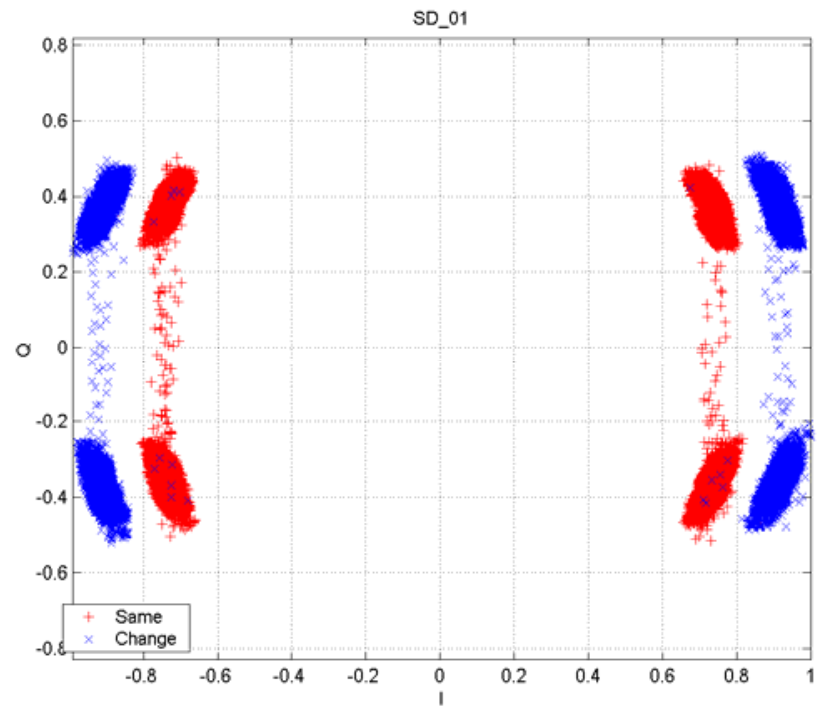

Figure 3 ISI separated by symbol sequence which provided sufficient separation for our purposes. There are two ways to determine $\alpha$ and $\beta$ :

1. Use only the inner blobs (which contributes the most to errors)

2. Find the EVM for the inner and outer blobs and average the two EVM measurements

Three methods of calibrating EVM to predict BER by determining the two coefficients were investigated; these were no added noise at RF, no added noise at IF and added noise at RF. Our setup allowed simultaneous EVM and BER measurements to remove any potential inaccuracies due to variation with time.

- $\quad$ The no added noise method uses a single EVM measurement at RF with a very high

$\mathrm{S} / \mathrm{N}$ and adjusts the coefficients for best fit to the BER curve.

- The no added noise at IF method used the same method as above, but the EVM measurement is at IF so any RF to IF distortion is captured. 
- The added noise method used simultaneous multiple RF EVM measurements and BER measurements with varying amounts of added noise with $\alpha$ and $\beta$ varied to obtain the best fit.

For reliability the satellite communications subsystem has duplicate hardware for each service and are called the primary and the redundant. To compare the effectiveness of the three methods, calibration based on the primary side of the communications subsystem was used to predict the BER from the redundant side for each link at the spacecraft level. The redundant sides generally had a slightly different curve; for example, the redundant side of the EMWIN link in Figure 2 had a $0.35 \mathrm{~dB}$ greater implementation margin than the primary side. As seen, EVM accurately predicted the difference in BER curves.

The results of the comparison of the three methods for the eight links tested are shown in Figure 4. All results were in agreement with measured values to within $0.5 \mathrm{~dB}$. The RF no added noise method, besides being much quicker, proved slightly more accurate than the other two methods. With one exception, the predictions based on the RF, no added noise method agreed with measurements to with $0.2 \mathrm{~dB}$ which is about the accuracy of the calibration and the BER measurements. We had expected that the added noise would be the best method because the additional measurements would provide more data points to fit the BER curve. Second best method was expected to be the IF no-added noise measurements as these would exclude additional distortion in the RF chain. The RF, no added noise was expected to be the poorest method. Instead, the results showed the opposite was true, the RF no added noise was best, the IF no added noise and finally, RF with added noise was the worst. One possible reason for this is that adding noise also adds another source of measurement error that is not present with the no added noise methods.

For integration and test, the RF no added noise method was used as this method was the simplest, and most accurate. However EVM measurements were made with added noise at the same time the BER measurements were made. The latter were used in post-launch testing described later in this paper.

Table 1 EVM measurements with and without adjacent channel interference

\begin{tabular}{|l|c|c|c|c|}
\hline \multicolumn{5}{|c|}{ Percent EVM } \\
\cline { 2 - 5 } & GOES-P & GOES-O \\
\cline { 2 - 5 } & $\begin{array}{c}\text { PMT No } \\
\text { ACI }\end{array}$ & $\begin{array}{c}\text { PMT with } \\
\text { ACI }\end{array}$ & $\begin{array}{c}\text { AMB1 No } \\
\text { ACI }\end{array}$ & with ACI \\
\hline DCPI01 & $1.65 \%$ & $2.20 \%$ & $2.16 \%$ & $2.29 \%$ \\
\hline DCPR01 & $2.75 \%$ & $2.85 \%$ & $2.37 \%$ & $2.34 \%$ \\
\hline EMWIN01 & $3.25 \%$ & $3.40 \%$ & $3.61 \%$ & $3.00 \%$ \\
\hline MDL01 & $3.70 \%$ & $3.75 \%$ & $3.52 \%$ & $3.49 \%$ \\
\hline PDR01 & $4.40 \%$ & $4.50 \%$ & $4.75 \%$ & $5.08 \%$ \\
\hline SAR04 & $4.15 \%$ & $4.25 \%$ & $4.06 \%$ & $4.22 \%$ \\
\hline SD02 & $3.45 \%$ & $3.45 \%$ & $4.78 \%$ & $4.93 \%$ \\
\hline WEFAX01 & $10.25 \%$ & $10.55 \%$ & $9.17 \%$ & $9.22 \%$ \\
\hline
\end{tabular}

In addition to saving time, EVM allowed measurements that would have been impossible with traditional BER methods which use pseudo-random number sequence. EVM allowed measurements of actual data with non pseudo-random structure including headers from instrument data packets on the satellite. As described in an adjacent paper ${ }^{6}$ EVM can identify the source of degradations, including intersymbol interference and phase

noise.

EVM cannot replace BER measurements for two reasons. First, a calibration against a BER test set is required. Second, if there is a large change in EVM due to a new, non-thermal degradation, EVM cannot accurately predict the BER. As an example, a large increase in phase noise (comparable to the thermal noise) would invalidate the calibration. The phase noise would be immediately apparent from a constellation plot. EVM would in this case detect the change in performance, but not accurately predict the new BER. A new calibration curve would be necessary to predict BER.

More commonly, EVM will vary over a narrow range, perhaps due to thermal variations. The methods described here allow a determination of the significance of that variation.

An example of the utility of EVM is determining if adjacent channel interference (ACI) degrades link quality. EVM measurements were made both with only the channel under test on and with all channels on with modulation. As shown in Table 1, the agreement of both methods showed that there was no degradation due to ACI. 
The benefits of EVM carried over to the post launch testing (PLT) which verified the usefulness of EVM measurements for PLT. Very good agreement with ground testing was obtained and will be discussed

\section{Test Setup \& Measurement Repeatability}

From the past experiences in BER measurements, it was found that the average measurement repeatability of BER is around $+/-0.3 \mathrm{~dB}$ and this accuracy is very difficult to maintain due to the difficulty of calibrating the test set-up. The conventional BER test on a spacecraft requires a modulator, up \& down converters (in most of cases), noise sources, additional filters, and a receiver. The EVM method introduced in this paper does not require noise sources, additional filters and a receiver to track performance. Furthermore, the latest VSA models support even higher frequencies up to X-band without requiring a downconverter. This eliminates a lot of the efforts required by BER measurement and increases measurement consistency significantly. The purpose of BER measurement in PLT is not only to perform the BER test in an on-orbit configuration but also to compare the result with the ground test results performed at the contractor's facilities, thus establishing a baseline to track satellite performance over life.

EVM is the only method to track the quality of service for the uncoded science data generated onboard the spacecraft. In commercial communications satellites, EVM is the only method of determining the quality of service of links where the link is uncoded or the coding is not known and there is no access to the uplink data.

One surprising aspect of VSAs is that they do not process data in the same way. One type of VSA finds the standard deviation of EVM for 10,000 points. To get the value for 60,000 points, 6 measurements of 10,000 points each are taken and the 6 are averaged. This gives a different (and incorrect) answer from another type of VSA which correctly finds the standard deviation for all 60,000 points.

\section{EVM Methods Compared}

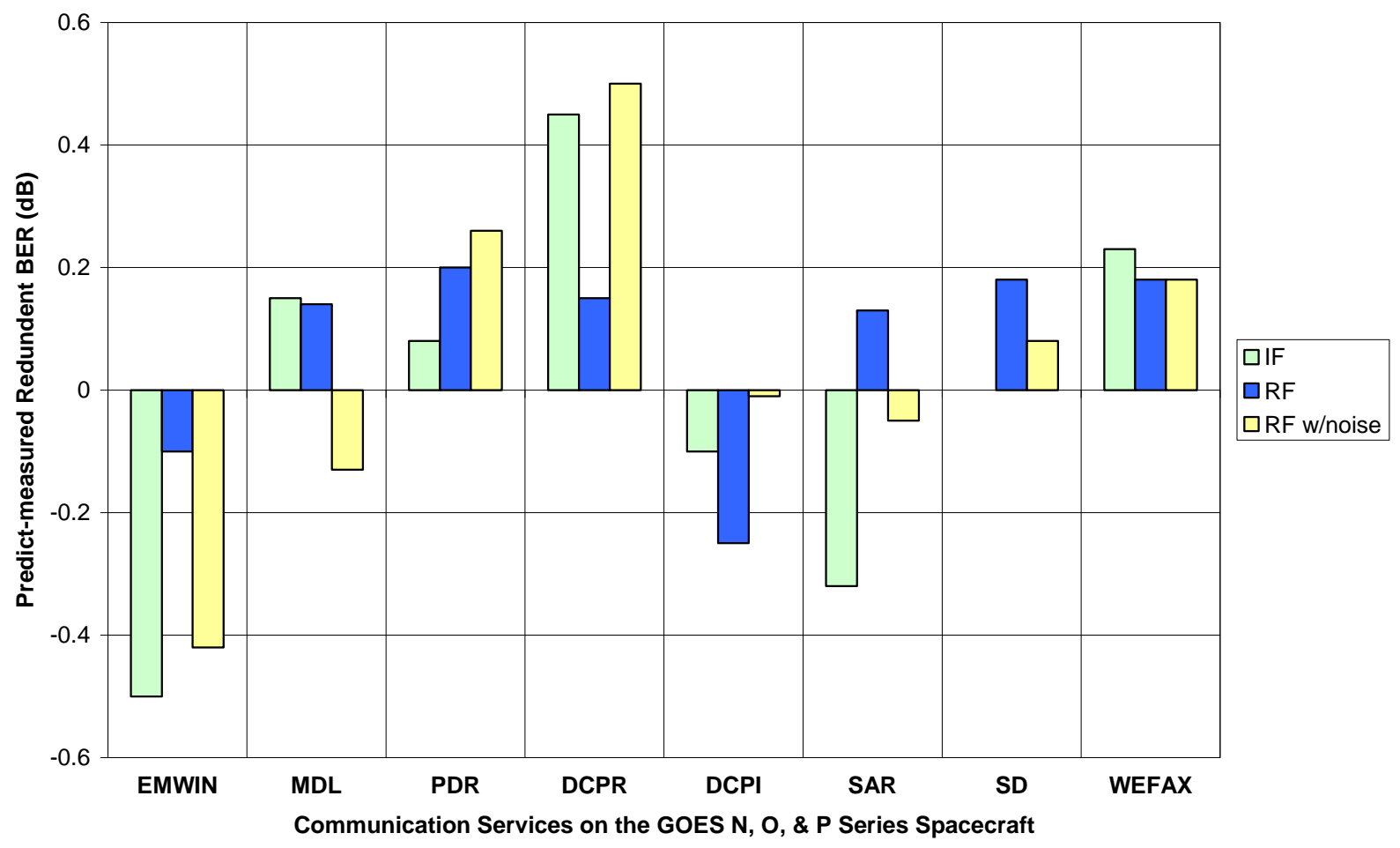

Figure 4 Three EVM calibration methods compared

\section{Analysis Methodology}

A one-time calibration of the EVM measurements is made by conducting simultaneous EVM and BER measurements at the start of Integration and Test (I\&T). There are two steps to this calibration, as the direct BER measurements are over a smaller Eb/No range (due to specification needs and time constraints). The first step is to measure BER and EVM over the range of Eb/No, and continue to measure EVM over a larger range of Eb/No. The 
added noise BER curve from EVM ( $\alpha 2$ and $\beta 2$ ) is generated. The second step is to generate a BER curve ( $\alpha 1$ and $\beta 1)$ from the no-added-noise EVM to the curve generated in the first step. Finally the no added noise BER curve is compared to the original BER measurement. Both sets of coefficients, $\alpha$ and $\beta$ are calculated using EXCEL's Solver to vary $\alpha$ and $\beta$ to find the minimum of the sum of the squares of the difference between the measured and predicted BER, as shown in Table 2. The starting points used by Solver sometimes must be varied to end up with the best curve.

The $\alpha 1$ and $\beta 1$ coefficients are used through the rest of the test program with the no added noise EVM measurement to generate the BER curves.

Table 2 Obtaining Calibration Coefficients

\begin{tabular}{|c|c|c|c|c|c|c|c|c|c|}
\hline & & & \\
\hline & $\mathrm{A}$ & B & $\mathrm{C}$ & D & $\mathrm{E}$ & $\mathrm{F}$ & $\mathrm{G}$ & $\mathrm{H}$ & $\mathrm{I}$ \\
\hline 1 & & & & & \multicolumn{2}{|c|}{ No Noise Added EVM } & \multicolumn{2}{|c|}{ Added-Noise EVM } & \\
\hline 2 & & & & & $\alpha 1$ & 2.2049 & $\alpha 2$ & 0.1298 & \\
\hline 3 & & & & & $\beta 1$ & 0.61901117 & $\beta 2$ & 0.424287703 & \\
\hline 4 & & & & & & & & \multirow{5}{*}{\begin{tabular}{|c|}
$\begin{array}{c}\text { Normalized } \\
\text { deviation } \\
\text { for No } \\
\text { Added } \\
\text { Noise Case }\end{array}$ \\
\end{tabular}} & \multirow{4}{*}{$\begin{array}{c}\text { Normalized } \\
\text { deviation } \\
\text { for Added } \\
\text { Noise case }\end{array}$} \\
\hline 5 & \multirow{3}{*}{\multicolumn{2}{|c|}{ Measured BER }} & \multicolumn{5}{|c|}{ EVM Calibration Example } & & \\
\hline 6 & & & \multirow{2}{*}{\multicolumn{2}{|c|}{$\begin{array}{l}\text { Calculated BER } \\
\text { from - No Noise } \\
\text { Added Case } \\
\end{array}$}} & \multirow{2}{*}{\multicolumn{3}{|c|}{$\begin{array}{l}\text { BER Curve fit from measured } \\
\text { BER and EVM - Added Noise Case }\end{array}$}} & & \\
\hline 7 & & & & & & & & & \\
\hline 9 & Eb/No & \begin{tabular}{|c|} 
BIT \\
ERROR \\
RATE(Pe)
\end{tabular} & $\begin{array}{c}\text { EVM } \\
(\%)\end{array}$ & BER (Pe) & Eb/No & EVM (\%) & BER (Pe) & & \\
\hline 10 & 10.035 & $4.02 \mathrm{E}-04$ & 6.52 & $2.973 \mathrm{E}-04$ & 10.035 & 30.94 & $3.001 \mathrm{E}-04$ & $9.28 \mathrm{E}-10$ & $3.05 \mathrm{E}-01$ \\
\hline 11 & 11.035 & $5.96 \mathrm{E}-05$ & 6.52 & $6.332 \mathrm{E}-05$ & 11.035 & 27.70 & $6.351 \mathrm{E}-05$ & $2.49 \mathrm{E}-02$ & $2.49 \mathrm{E}-02$ \\
\hline 12 & 12.035 & $9.26 \mathrm{E}-06$ & 6.52 & $1.039 \mathrm{E}-05$ & 12.035 & 25.11 & $1.061 \mathrm{E}-05$ & $-1.41 \mathrm{E}-01$ & $-1.41 \mathrm{E}-01$ \\
\hline 13 & 13.035 & $1.26 \mathrm{E}-06$ & 6.52 & $1.290 \mathrm{E}-06$ & 13.035 & 22.75 & $1.306 \mathrm{E}-06$ & $-7.28 \mathrm{E}-02$ & $-7.28 \mathrm{E}-02$ \\
\hline 14 & 14.035 & $1.29 \mathrm{E}-07$ & 6.52 & $1.203 \mathrm{E}-07$ & 14.035 & 20.67 & $1.152 \mathrm{E}-07$ & $6.82 \mathrm{E}-02$ & $4.65 \mathrm{E}-03$ \\
\hline 16 & & & & & & & & & \\
\hline 17 & & & & & & & $\begin{array}{l}\text { SUM OF } \\
\text { SQUARES }\end{array}$ & $9.17618 \mathrm{E}-02$ & 1.02699E-01 \\
\hline 18 & \multirow{2}{*}{\multicolumn{2}{|c|}{$\begin{array}{c}\text { EXAMPLE BER } \\
\text { from EVM }(6.9 \%)\end{array}$}} & & & & & & & \\
\hline 19 & & & & & & & & & \\
\hline 20 & Eb/No & \begin{tabular}{|l|} 
BER (Pe) \\
\end{tabular} & & & & & & & \\
\hline 21 & 10.035 & $3.566 \mathrm{E}-04$ & \multirow{5}{*}{\multicolumn{4}{|c|}{$\begin{array}{l}\text { In this example, the coefficients } \alpha_{1} \& \beta_{1} \text { from } \\
\text { the No Noise Added case are used }\end{array}$}} & & & \\
\hline 22 & 11.035 & $7.985 \mathrm{E}-05$ & & & & & & & \\
\hline 23 & 12.035 & $1.398 \mathrm{E}-05$ & & & & & & & \\
\hline 24 & 13.035 & $1.885 \mathrm{E}-06$ & & & & & & & \\
\hline 25 & 14.035 & \begin{tabular}{|l|}
$1.949 \mathrm{E}-07$ \\
\end{tabular} & & & & & & & \\
\hline
\end{tabular}

\section{Post Launch Testing with EVM}

The coefficient sets calibrated on the GOES-O \& P spacecrafts for each service had to be used for GOES-N, (which was renamed GOES 13 once in service) PLT as there was no ground EVM calibration data for GOES-13. Despite this, the measured bit error rates trended well with BER predicted from EVM measurements, within a few tenths of a dB.

There is no way to take a noise free measurement of EVM in PLT as the link adds a substantial amount of noise. Therefore we used the coefficients derived from the added noise EVM measurements to generate a BER vs. Eb/No curve. From a single EVM measurement and knowing Eb/No, a BER vs. Eb/No was generated. From this curve, the BER at the specified Eb/No was found and specification performance verified. The bit error rate estimation from the EVM measurement in this example clearly shows this method can closely and consistently estimate the bit error rate results within a bit error rate measurement repeatability and uncertainty boundaries. For one way links, e.g. 
links where a varying data pattern is generated on the spacecraft, the only way to get BER performance is with EVM as there was no way to have knowledge of the flight bit pattern.

GOES - BER Ground Measured and predicted BER from PLT EVM measurements using GOES O and $P$ coefficients

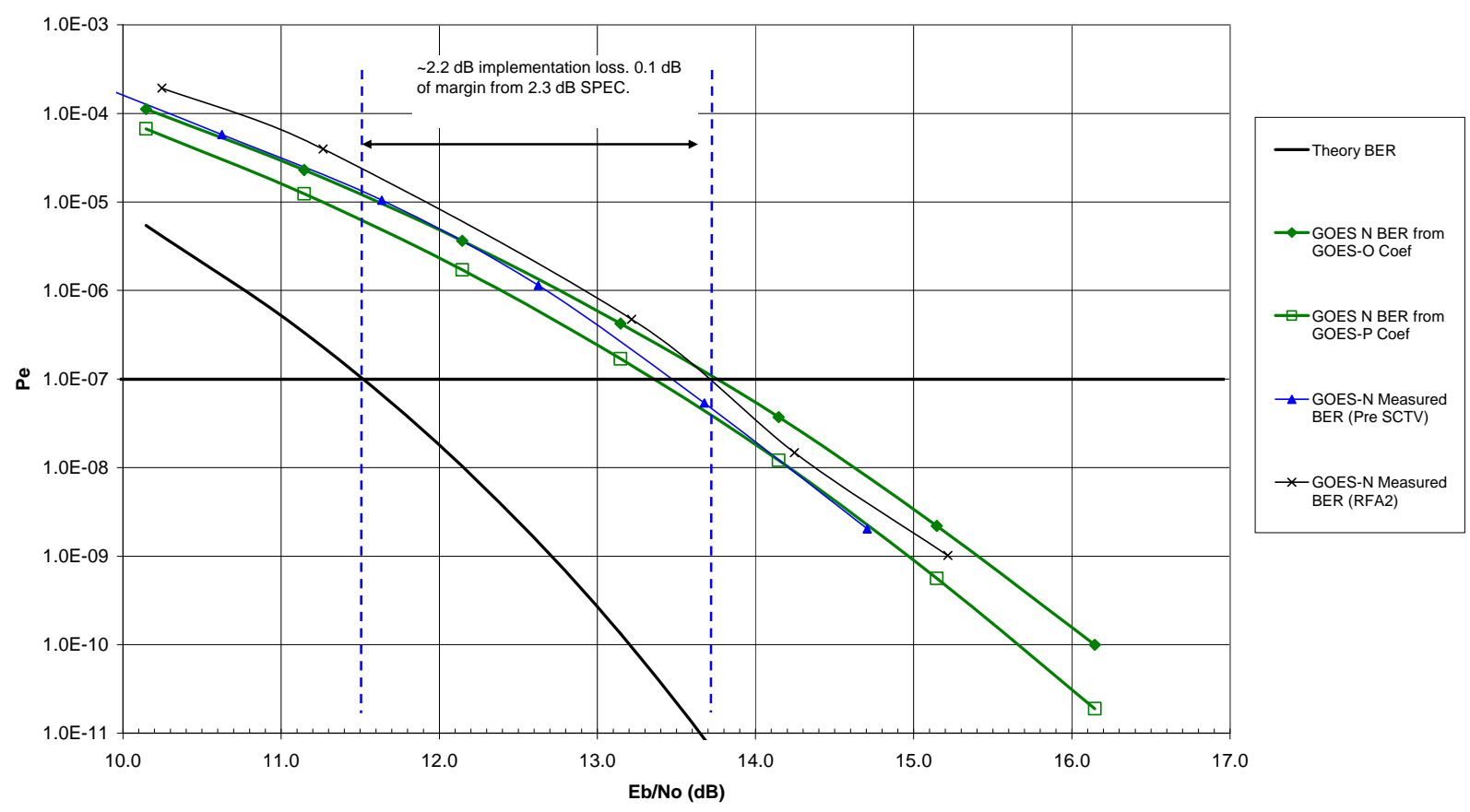

Figure 5 BER trending on GOES 13 using coefficients on GOES $O$ and $P$

\section{Conclusions}

While EVM provides a very simple and quick method for estimating the bit error rate of a communication channel, there are several limitations need to be considered carefully during the calibration and the measurement.

- Changing any hardware after the point at which EVM is measured could invalidate the calibration since EVM measures the quality of the link up to the point where the VSA is connected and not past that point.

- If there are substantial changes in the EVM values, EVM may not accurately predict the new BER based on the prior calibration. For example, if phase noise suddenly degrades performance EVM increases dramatically and so will the link degradation. The new measure must be evaluated based on the range of the calibration. But either way, a big variation from expected EVM should lead to more investigation.

- Vector Signal Analyzer setup: Use consistent instrument setup parameters. The instrument setup parameters should be well maintained and controlled during test phases to avoid any possible measurement errors introduced by inconsistent configuration of the instrument. We recorded VSA setup parameters for each modulation format on a disk and used the same settings throughout the test program.

- EVM can not detect errors that occur outside of the short measurement time (infrequent burst errors).

- For PLT or other applications where there is a substantial contribution from thermal noise, the noise added method should be used. Where Eb/No is high, the no added noise case should be used.

- If the two blob effect (ISI) is seen in the VSA, the EVM data must be processed to separate out the EVM for each blob.

\footnotetext{
${ }^{1}$ Joao L. Pinto and Izzat Darwazeh, Phase Distortion and Error Vector Magnitude for 8-PSK Systems published in Proceedings ConfeTele 2001 available at http://www.co.it.pt/conftele2001/proc/pap098.pdf
} 
${ }^{2}$ R. Haswsun, M. Flaherty, R. Matreci, M. Taylor, Effective Evaluation of Link Quality Using Error Vector Magnitude Techniques, 1997 Wireless Communications Conference at

http://ieeexplore.ieee.org/iel3/4903/13517/00622254.pdf?arnumber=622254

${ }^{3}$ S. Forestier, B. Bouysse, R. Quere, A. Mallet, J.M. Nebus, L.Lapierre, A Dynamic Bias Technique of PHEMT SSPA for Optimized PAE and EVM applied MQAM Satellite Communication Systems, $33^{\text {rd }}$ European Microwave Conference (Munich 2003)

${ }^{4}$ Private communication with D. Fisher, GSFC, NASA

${ }^{5}$ Kent K. Johnson. Residual BER Prediction on the Web at:

http://www.home.agilent.com/upload/cmc_upload/All/06-27-01_PredictingResidualBER-1-Johnson_503NOTES.pdf

${ }^{6}$ An alternative equation was presented by one of the authors (R. Peters) at the 23trdAIAA International Communications Satellite Systems Conference in an unpublished paper, Using EVM to Determine BER on a

Satellite Link

${ }^{7}$ Carmen Kocinski, Using the EVM to predict BER performance of QPSK modulators with phase noise and timing jitter impairment this conference

${ }^{8}$ Hassun, R. Flaherty, M. Matreci, R. Taylor, M. "Effective Evaluation of Link Quality Using Error Vector Magnitude Techniques". Wireless Communications Conference, 1997. pp. 89-94. 